\title{
Cold-adapted bacterial extracts as source of anti-infective and antimicrobial compounds against Staphylococcus aureus
}

\author{
Marco Artini ${ }^{\ddagger}, 1$, Rosanna Papa ${ }^{\ddagger 1}$, Gianluca Vrenna ${ }^{1}$, Concetta Lauro², Annarita \\ Ricciardelli², Angela Casillo², Maria M Corsaro², Maria L Tutino², Ermenegilda Parrilli² \& \\ Laura Selan*,1 \\ ${ }^{1}$ Department of Public Health \& Infectious Diseases, Sapienza University, 00185 Rome, Italy \\ ${ }^{2}$ Department of Chemical Sciences, Federico II University, 80126 Naples, Italy \\ *Author for correspondence: Tel.: +39 0649694 261; Fax: +39 0649694 298; laura.selan@uniroma1.it \\ $\ddagger$ Authors contributed equally
}

\begin{abstract}
Aim: The dramatic emergence of antibiotic resistance has directed the interest of research toward the discovery of novel antimicrobial molecules. In this context, cold-adapted marine bacteria living in polar regions represent an untapped reservoir of biodiversity endowed with an interesting chemical repertoire. The aim of this work was to identify new antimicrobials and/or antibiofilm molecules produced by coldadapted bacteria. Materials \& methods: Organic extracts obtained from polar marine bacteria were tested against Staphylococcus aureus. Most promising samples were subjected to suitable purification strategies. Results: Results obtained led to the identification of a novel lipopeptide able to effectively inhibit the biofilm formation of $S$. aureus. Conclusion: New lipopeptide may be potentially useful in a wide variety of biotechnological and medical applications.
\end{abstract}

First draft submitted: 17 May 2019; Accepted for publication: 22 August 2019; Published online: 9 October 2019

Keywords: antibacterial $\bullet$ antibiofilm $\bullet$ antimicrobial $\bullet$ lipopeptide $\bullet$ organic extract $\bullet$ polar bacteria $\bullet$ Pseudomonas - Staphylococcus aureus

In recent years, bacterial infections represent a serious challenge for human health due to the widespread diffusion of resistance to antibiotics, since more than $70 \%$ of pathogenic bacteria display resistance to at least one antibiotic commonly used in therapy [1]. This feature is often related to the ability to form biofilm [2], a phenotypic lifestyle that renders bacteria highly tolerant to exogenous stressors, including antibiotics or other biocides.

Staphylococci represent a prevalent cause of chronic infections associated to biofilm phenotype [3]; Staphylococcus aureus in particular, deploying a wide array of virulence factors, can acquire resistance to the majority of antibacterial compounds. This feature makes $S$. aureus a 'superbug' further reinforced by continuous emergence of new clones [4].

S. aureus can be frequently isolated as a commensal of the human body, colonizing districts of healthy individuals such as skin and mucous membranes, including nares and guts. S. aureus can be persistently isolated from the nasal cavity of $20 \%$ of healthy individuals, while $30 \%$ are intermittent carriers. This colonization represents an important reservoir of this pathogen, and significantly increases the chances of infections [4].

Within the past two decades, the continuous and rapid emergence of antibiotic resistance has directed the interest of research toward the discovery of novel antibiofilm compounds and antimicrobial molecules [5]. In the literature, there are several reports about the novel synthesis of biomaterials effective against $S$. aureus and able to combat drug resistance phenomenon [6].

Recently, many scientists are analyzing natural compounds extracted from plants and marine microorganisms, in order to identify new and effective antibiofilm molecules [7-10]. Indeed, the bioprospecting of natural environments has allowed to discover, produce and commercialize many new antibiotics [11] and offers novel key scaffolds for drug development [12].

Future Medicine 
Table 1. Strains used in this study.

\begin{tabular}{|c|c|c|}
\hline Strain & Origin & Ref. \\
\hline P. haloplanktis TAE79 & $\begin{array}{l}\text { Antarctic seawater }{ }^{\dagger} \text { (algae necrosized suspended in } \\
\text { seawater) }\end{array}$ & Liège collection \\
\hline P. haloplanktis TAE80 & $\begin{array}{l}\text { Antarctic seawater }{ }^{\dagger} \text { (algae necrosized suspended in } \\
\text { seawater) }\end{array}$ & Liège collection \\
\hline Pseudomonas sp. TAD1S & Antarctic seawater ${ }^{\dagger}$ & Liège collection \\
\hline Pseudomonas sp. TAD18 & Antarctic seawater ${ }^{\dagger}$ (frozen algae) & Liège collection \\
\hline Pseudomonas sp. TAA207 & Antarctic seawater ${ }^{\dagger}$ (marine sediment) & Liège collection \\
\hline Psychromonas arctica & Arctic seawater (Svalbard islands, Arctic) & [53] \\
\hline P. haloplanktis TAB23 & Antarctic seawater ${ }^{\dagger}$ & [54] \\
\hline P. haloplanktis TAE56 & $\begin{array}{l}\text { Antarctic seawater }{ }^{\dagger} \text { (algae necrosized suspended in } \\
\text { seawater) }\end{array}$ & Liège collection \\
\hline P. haloplanktis TAE57 & $\begin{array}{l}\text { Antarctic seawaterr }{ }^{\dagger} \text { (algae necrosized suspended in } \\
\text { seawater) }\end{array}$ & Liège collection \\
\hline S. aureus $6538 \mathrm{P}$ & Clinical isolate, reference strain for antimicrobial tests & ATCC collection \\
\hline S. aureus 20372 & Clinical isolate & ATCC collection \\
\hline S. aureus 25923 & Clinical isolate, reference strain for antimicrobial tests & ATCC collection \\
\hline
\end{tabular}

Marine microorganisms are considered an inexhaustible source for the discovery of new antimicrobial compounds, since they must survive in an environment characterized by climatic variations and compete with others for space and nutrition. Several biologically active compounds (such as antifouling, anticancer, antimicrobial) have been obtained from marine sources [13]. Some of them are already investigated for the treatment of various diseases [14-16].

In this context, cold-adapted marine bacteria deriving from polar regions represent an unexploited reservoir of biodiversity provided with an attracting chemical repertoire. Unusual survival strategies have been developed by bacteria living in prohibitive environmental conditions, according to an antagonistic behavior mandatory offering competitive advantages due to the limitation of the growth of other microorganisms [17]. When procuration of nutrients is limited or difficult, this behavior offers a growth advantage; in this view, Antarctic microorganisms could have developed peculiar characteristics, such as chemically complex metabolites, that guarantee survival in extreme habitats [18]. Some molecules obtained from these bacteria display antifouling, antimicrobial and other activities interesting for possible pharmaceutical applications [19]. We recently reported that Pseudoalteromonas haloplanktis TAC125, an Antarctic marine bacterium, produce and secrete many different compounds of biotechnological interest. These compounds include molecules that inhibit biofilm growth by Staphylococcus epidermidis, a saprophyte of the human skin acting as a pathogen after colonization of artificial medical devices [20-23], and antimicrobial compounds that inhibit the growth of Burkholderia cepacia complex (Bcc), organisms that pose a significant health risk to cystic fibrosis patients [24,25]. Furthermore, cell-free supernatants obtained from different bacterial cultures of polar marine bacteria showed interesting antibiofilm activities on S. epidermidis, Pseudomonas aeruginosa and $S$. aureus [26].

The aim of this paper was the identification of new antimicrobials and/or antibiofilm molecules produced by bacteria adapted to cold habitats. Intracellular and extracellular organic extracts obtained from polar marine bacteria, such as Psychromonas, Pseudomonas and Pseudoalteromonas genera, have been tested against S. aureus. Most promising samples were subjected to suitable purification strategies in order to identify molecules responsible for the sought biological activity.

\section{Materials \& methods}

\section{Bacterial strains \& culture conditions}

Bacterial strains used in this work are reported in Table 1. Polar bacteria were grown in flasks in synthetic medium GG (D-gluconic acid sodium $10 \mathrm{~g} / \mathrm{l}$, L-glutamic acid $10 \mathrm{~g} / \mathrm{l}, \mathrm{NaCl} 10 \mathrm{~g} / \mathrm{l} ; \mathrm{NH}_{4} \mathrm{NO}_{3} 1 \mathrm{~g} / 1 ; \mathrm{KH}_{2} \mathrm{PO}_{4} \cdot 7 \mathrm{H}_{2} \mathrm{O}$ $\left.1 \mathrm{~g} / \mathrm{l} ; \mathrm{MgSO}_{4} \cdot 7 \mathrm{H}_{2} \mathrm{O} 200 \mathrm{mg} / \mathrm{l} ; \mathrm{FeSO}_{4} \cdot 7 \mathrm{H}_{2} \mathrm{O} 5 \mathrm{mg} / \mathrm{l} ; \mathrm{CaCl}_{2} \cdot 2 \mathrm{H}_{2} \mathrm{O} 5 \mathrm{mg} / \mathrm{l}\right)$ at $15^{\circ} \mathrm{C}$ under vigorous agitation (180 r.p.m) until stationary phase (72-90 h).

S. aureus was grown in Brain Heart Infusion (BHI) broth (Oxoid, Basingstoke, UK) at $37^{\circ} \mathrm{C}$. Culture broths were performed under vigorous agitation $(180 \mathrm{rpm})$, while biofilm formation was assessed in static conditions. 
Bacterial strains grown in $\mathrm{BHI}$ broth were kept at $-80^{\circ} \mathrm{C}$ with glycerol at a final concentration of $15 \%$.

\section{Organic extract preparation}

Polar bacterial cultures previously described were collected and subjected to a liquid-liquid extraction as an initial separation technique to process the cell-free supernatants and the cellular pellets, to obtain 'extracellular extracts' and 'intracellular extracts', respectively.

Cell-free supernatants and cell pellets were recovered by centrifugation at 6000 r.p.m at $4^{\circ} \mathrm{C}$ for $50 \mathrm{~min}$. After centrifugation, the supernatants were separated from the cells and were sterilized by filtration through membranes with a pore diameter of $0.22 \mu \mathrm{m}$. Cell pellets and cell-free supernatants were frozen at $-80^{\circ} \mathrm{C}$ until use.

Liquid-liquid extraction was performed on cell pellets and cell-free supernatants separately, without adding cryoprotectants. In detail, cell pellets and supernatants were thawed, then cell pellets were resuspended in an opportune volume of sterile distilled water. Obtained samples were stirred with ethyl acetate in a volume ratio of 2:1 (assay percent range $\geq 99.5 \%$ ) (Sigma-Aldrich, St. Louis, MO, US) and mixed at $1 \%$ with acid formic (assay percent range $=90 \%$; JT Baker, Munich, Germany). Each solution was stirred at least for $30 \mathrm{~min}$ and then centrifuged at 3000 r.p.m for $30 \mathrm{~min}$. The resulting two phases were separated, and the organic phase was recovered and dried using a rotary evaporator Rotavapor (Buchi R-210) at a temperature lower than $40^{\circ} \mathrm{C}$. The resulting organic extracts were aliquoted in $4 \mathrm{mg}$ dry-weight samples and stored at $-20^{\circ} \mathrm{C}$.

\section{Fractionation of the intracellular extract of TAA207 \& TAD1S}

The intracellular extracts (named IN_) from Pseudomonas sp. TAA 207 (IN_TAA207, $100 \mathrm{mg}$ ) and from Pseudomonas sp. TAD1S (IN_TAD1S, $113 \mathrm{mg}$ ) were both fractionated on silica gel columns. The columns $(30 \mathrm{ml}$, $50 \times 0.7 \mathrm{~cm}$ for IN_TAA207 and $49 \mathrm{ml}, 32 \times 0.7 \mathrm{~cm}$ for IN_TAD1S, respectively) were initially eluted with $\mathrm{CHCl}_{3}: \mathrm{CH}_{3} \mathrm{OH}$ ranging from 100 to $70 \%$ of $\mathrm{CHCl}_{3}$, and then with $\mathrm{CHCl}_{3}: \mathrm{CH}_{3} \mathrm{OH}: \mathrm{H}_{2} \mathrm{O} 9 / 3 / 0.5 \mathrm{v} / \mathrm{v} / \mathrm{v}$. Only for IN_TAD1S, a further elution with $\mathrm{CHCl}_{3}: \mathrm{CH}_{3} \mathrm{OH}: \mathrm{H}_{2} \mathrm{O}$ 6/4/0.5 v/v/v was performed.

The fractions resulted to be active against $S$. aureus (IN_ TAA207: fraction 3; and IN_TAD1S: fractions 5-8-1120, respectively) were acetylated and analyzed by GC-MS technique. In detail, fraction 3 from IN_ TAA207 was analyzed on an Agilent Technologies gas chromatograph 6850A equipped with a mass selective detector 5973N and a Zebron ZB-5 capillary column (Phenomenex, $30 \mathrm{~m} \times 0.25 \mathrm{~mm}$ i.d., flow rate $1 \mathrm{ml} / \mathrm{min}$, He as carrier gas); fractions 5-8-11-20 from IN_TAD1S were analyzed on a Agilent Technologies gas chromatograph 7820A equipped with a mass selective detector 5977Band a HP-5 capillary column (Agilent, $30 \mathrm{~m} \times 0.25 \mathrm{~mm}$ i.d., flow rate $1 \mathrm{ml} / \mathrm{min}$, He as carrier gas). All derivatives were analyzed by using the following temperature program: $150^{\circ} \mathrm{C}$ for $3 \mathrm{~min}$, from 150 to $300^{\circ} \mathrm{C}$ at $15^{\circ} \mathrm{C} / \mathrm{min}$, at $300^{\circ} \mathrm{C}$ for $5 \mathrm{~min}$.

The same purification procedure was used for EX_TAD1S $(70 \mathrm{mg})$. The silica gel column $(46 \mathrm{ml}, 30 \times 0.7 \mathrm{~cm})$ was eluted with the same solvent mixtures used for IN_TAD1S. Fraction 15, active against $S$. aureus USA300, was analyzed by ${ }^{1} \mathrm{H}$ NMR spectrum performed by using a Bruker Avance-DRX $600 \mathrm{MHz}$ spectrometer equipped with a cryoprobe. The spectrum was acquired at $298 \mathrm{~K}$ in $\mathrm{CDCl}_{3}: \mathrm{CD}_{3} \mathrm{OD} 3: 1$.

\section{Minimal inhibitory concentration}

The antimicrobial activity of each extract was evaluated. The minimal inhibitory concentration (MIC) was defined as the lowest concentration of antimicrobial that yields at least $99.9 \%$ reduction (i.e., three log-units) of bacterial growth compared with the untreated bacteria as reported by Clinical \& Laboratory Standards Institute (CLSI) and National Committee for Clinical Laboratory Standards (NCCLS) methods. MIC was determined in duplicate by twofold serial dilution (CLSI, 2017), as reported [27].

The wells of a sterile 48-well flat-bottomed polystyrene plate were filled with $400 \mu \mathrm{l}$ of $1 / 100$ diluted overnight bacterial cultures grown in BHI. Each extract was tested starting from a concentration of $4 \mathrm{mg} / \mathrm{ml}$, as reported in the literature [28]. After overnight incubation at $37^{\circ} \mathrm{C}$, the antimicrobial activity was optically evaluated comparing treated and untreated samples.

\section{Biofilm formation by $S$. aureus}

Biofilm formation by $S$. aureus was evaluated in the presence of organic extracts. Quantification of in vitro biofilm production was based on a method previously reported [29]. Briefly, the wells of a sterile 96-well flat-bottomed polystyrene plate were filled with $100 \mu \mathrm{l}$ of BHI broth. 1/100 dilution of overnight bacterial cultures was added 
into each well. Dried extracts were first dissolved in DMSO at an opportune concentration, then diluted in BHI before use.

The first row of the multiwell contained the untreated bacteria, while each of the remaining rows contained serial dilutions of the organic extract, starting from the second row.

After the inoculation, the plates were incubated aerobically for $20 \mathrm{~h}$ at $37^{\circ} \mathrm{C}$ in static condition. Biofilm formation was measured by crystal violet staining. After treatment, culture broths were gently removed; each well was washed three-times with water and patted dry on a piece of paper towel in an inverted position. Each well was stained with $0.1 \%$ crystal violet (incubation at room temperature for $15 \mathrm{~min}$ ). To remove the excess of dye, each well was washed three-times with water, and thoroughly dried. The remaining dye, bound to biofilm, was solubilized with $80 \%(\mathrm{v} / \mathrm{v})$ ethanol and $20 \%(\mathrm{v} / \mathrm{v})$ glacial acetic acid. After $30 \mathrm{~min}$ of incubation at room temperature, (optical density) OD at $590 \mathrm{~nm}$ was measured to quantify the total biomass of biofilm formed in each well. Each data point is composed of three independent experiments each performed at least in six replicates.

\section{Statistics \& reproducibility of results}

Data reported were statistically validated using Student's $t$-test comparing the mean absorbance of treated and untreated samples. The significance of differences between mean absorbance values was calculated using a twotailed Student's $t$-test. A p-value of less than 0.05 was considered significant.

\section{Results}

\section{Setup of two libraries from polar bacterial cultures}

A selection of nine polar marine bacteria (Table 1) were grown, as described in the Materials \& methods section. The synthetic minimal medium named D-gluconic and L-glutamic acid medium (GG) [25] was chosen to minimize the potential interference of culture medium on the organic extraction step due to reduction of the sample complexity, and to exploit the biosynthetic potential of bacterial metabolism. Furthermore, the choice of a minimum medium should promote in the bacterial cell the activation of alternative metabolic pathway that could maximize the production of secondary metabolites. The whole bacterial cultures were then recovered and separately extracted (cell pellet and supernatant) by using ethyl acetate. It is often adopted for the downstream recovery of fermentation products such as antibiotics or secondary metabolite.

In this way, for each bacterial strain, we obtained an intracellular extract (IN_StrainName) and an extracellular one (EX_StrainName).

\section{Screening of antimicrobial activities of extracts against $S$. aureus}

Intracellular and extracellular extracts were analyzed for their antimicrobial activity against $S$. aureus 6538P. The MIC of extracts is reported in Table 2. A discrete number of selected extracts showed antimicrobial activity against S. aureus 6538P. In detail, IN_TAB23, EX_TAE56 and EX_TAE57 showed antimicrobial activity at a concentration higher than $1 \mathrm{mg} / \mathrm{ml}$.

\section{Screening of antibiofilm activity of intracellular extracts against $S$. aureus}

Intracellular extracts were also screened for their ability to inhibit biofilm formation of $S$. aureus 6538P. Several extracts were able to inhibit biofilm formation (Figure 1). Considering that IN_TAB23 displayed an antimicrobial activity at a concentration of $1 \mathrm{mg} / \mathrm{ml}$ (Table 2), it was tested for antibiofilm activity at a concentration of $0.5 \mathrm{mg} / \mathrm{ml}(1 / 2 \mathrm{MIC}$ value). Results obtained showed that this extract also possessed an antibiofilm activity at sub-MIC concentration. Best results were obtained with IN_TAA207 and IN_TAD1S extracts and, for this reason, they were selected for further analyses.

Experiments were performed on three different $S$. aureus strains, characterized by qualitative and quantitative differences in biofilm composition [29], using serial dilutions of each extract, starting from a concentration of $4 \mathrm{mg} / \mathrm{ml}$ (Figure 2). Figure 2A describes the activity of IN_TAA207 on biofilm formation of S. aureus 6538P, 20372 and 25923 strains. A strong inhibitory effect was confirmed for all tested strains, despite their differences in biofilm composition. Furthermore, inhibition of biofilm formation is clearly dose-dependent, starting from a reduction of about $40 \%$ at a concentration of $0.5 \mathrm{mg} / \mathrm{ml}$.

As shown in Figure 2B, IN_TAD1S extract reduced biofilm production of all three tested $S$. aureus strains, with different extent. However, in these experiments, a clear dose-dependent effect was not observed. 
Table 2. Minimal inhibitory concentration of organic extracts from bacterial supernatants and whole cells on S. aureus 6538P.

\begin{tabular}{|c|c|}
\hline Extract & S. aureus 6538P \\
\hline \multicolumn{2}{|c|}{ Intracellular extracts } \\
\hline IN_TAE79 & $>4 \mathrm{mg} / \mathrm{ml}$ \\
\hline IN_TAE80 & $>4 \mathrm{mg} / \mathrm{ml}$ \\
\hline IN_TAD1S & $>4 \mathrm{mg} / \mathrm{ml}$ \\
\hline IN_TAD18 & $>4 \mathrm{mg} / \mathrm{ml}$ \\
\hline IN_TAA207 & $>4 \mathrm{mg} / \mathrm{ml}$ \\
\hline IN_ARTICA & $>4 \mathrm{mg} / \mathrm{ml}$ \\
\hline IN_TAB23 & $1 \mathrm{mg} / \mathrm{ml}$ \\
\hline IN_TAE56 & $>4 \mathrm{mg} / \mathrm{ml}$ \\
\hline IN_TAE57 & $>4 \mathrm{mg} / \mathrm{ml}$ \\
\hline \multicolumn{2}{|c|}{ Supernatant extracts } \\
\hline EX_TAE79 & $>4 \mathrm{mg} / \mathrm{ml}$ \\
\hline EX_TAE80 & $>4 \mathrm{mg} / \mathrm{ml}$ \\
\hline EX_TAD1S & $>4 \mathrm{mg} / \mathrm{ml}$ \\
\hline EX_TAD18 & $>4 \mathrm{mg} / \mathrm{ml}$ \\
\hline EX_TAA207 & $>4 \mathrm{mg} / \mathrm{ml}$ \\
\hline EX_ARTICA & $>4 \mathrm{mg} / \mathrm{ml}$ \\
\hline EX_TAB23 & $>4 \mathrm{mg} / \mathrm{ml}$ \\
\hline EX_TAE56 & $1 \mathrm{mg} / \mathrm{ml}$ \\
\hline EX_TAE57 & $1 \mathrm{mg} / \mathrm{ml}$ \\
\hline
\end{tabular}

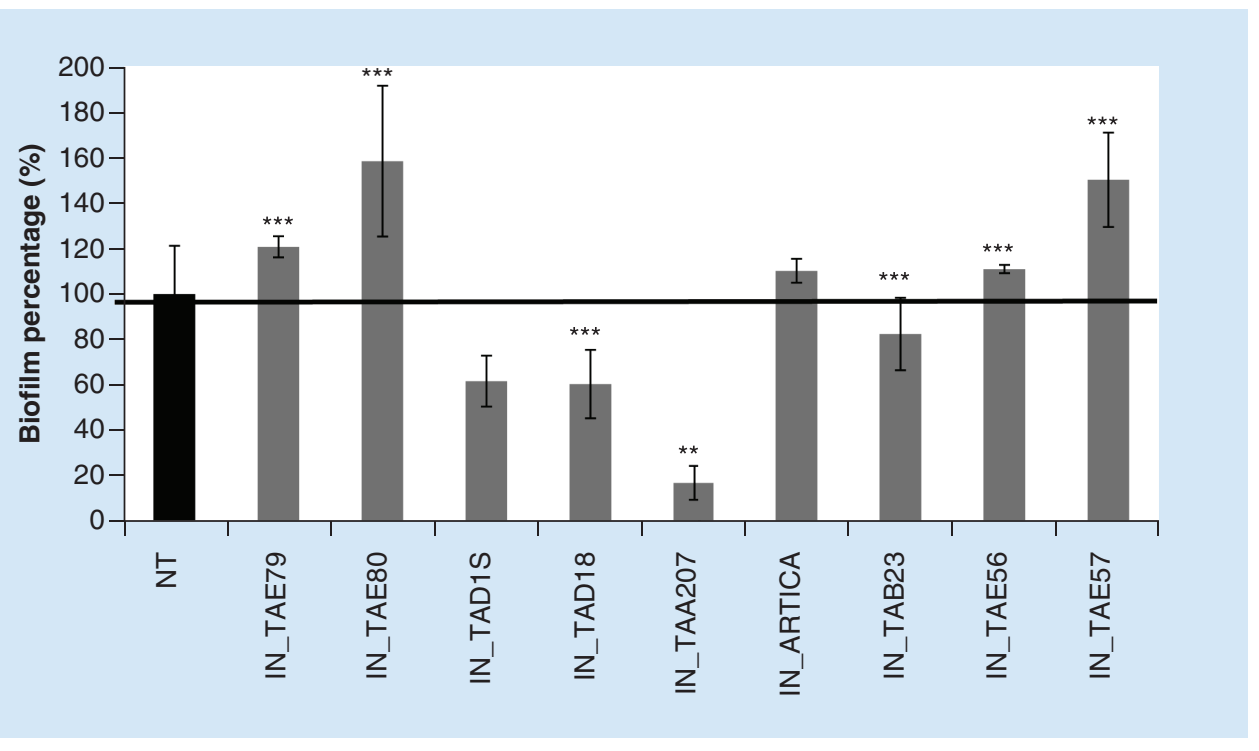

Figure 1. Intracellular extracts from cold-adapted bacteria on S. aureus 6538P biofilm formation. Data are reported as the percentage of residual biofilm after the treatment. Each data point represents the mean \pm SD of five independent samples. Differences in mean absorbance were compared with the untreated control (NT, black bar) and considered significant when $\mathrm{p}<0.05$ according to Student's $t$-test.

${ }^{*} \mathrm{p}<0.05 ; * * \mathrm{p}<0.01 ; * * * \mathrm{p}<0.001$.

NT: Not treated; SD: Standard deviation.

Fractionation of intracellular extracts from Pseudomonas sp. TAA207 \& from Pseudomonas sp.

TAD1S

IN_TAA207 and IN_TAD1S were fractionated on a silica gel column as reported in the Methods section (Figure 3).

The fractions obtained from IN_TAA207 were tested, at a concentration of $200 \mu \mathrm{g} / \mathrm{ml}$, to evaluate the antibiofilm 
(A)

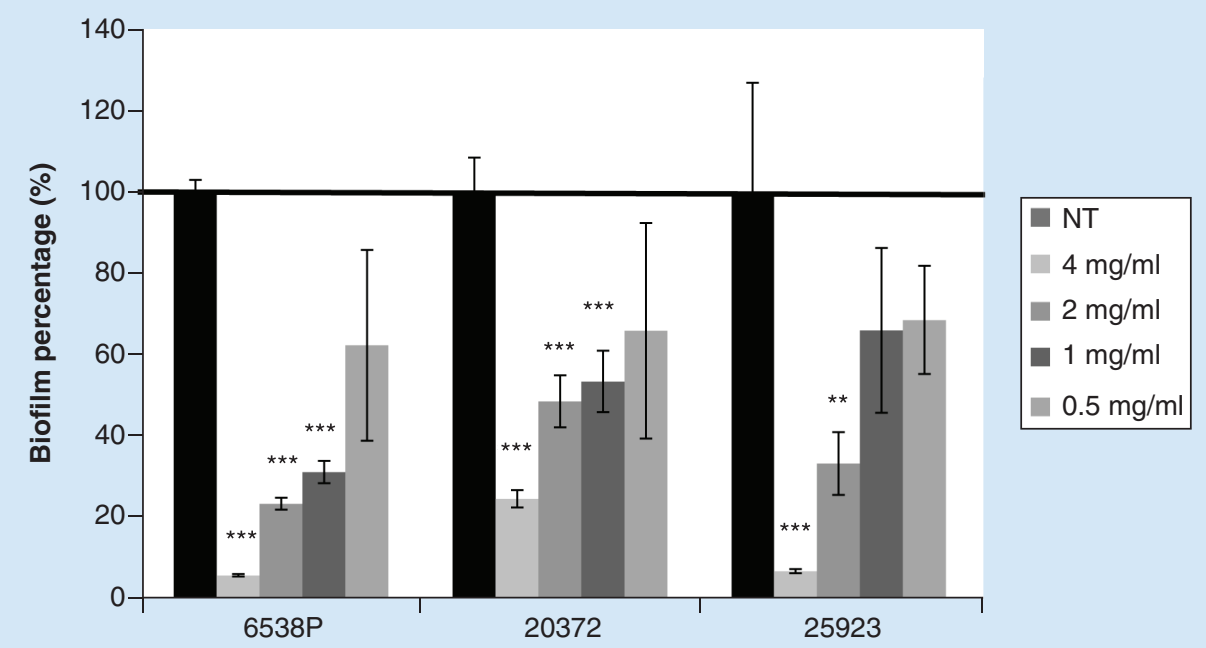

(B)

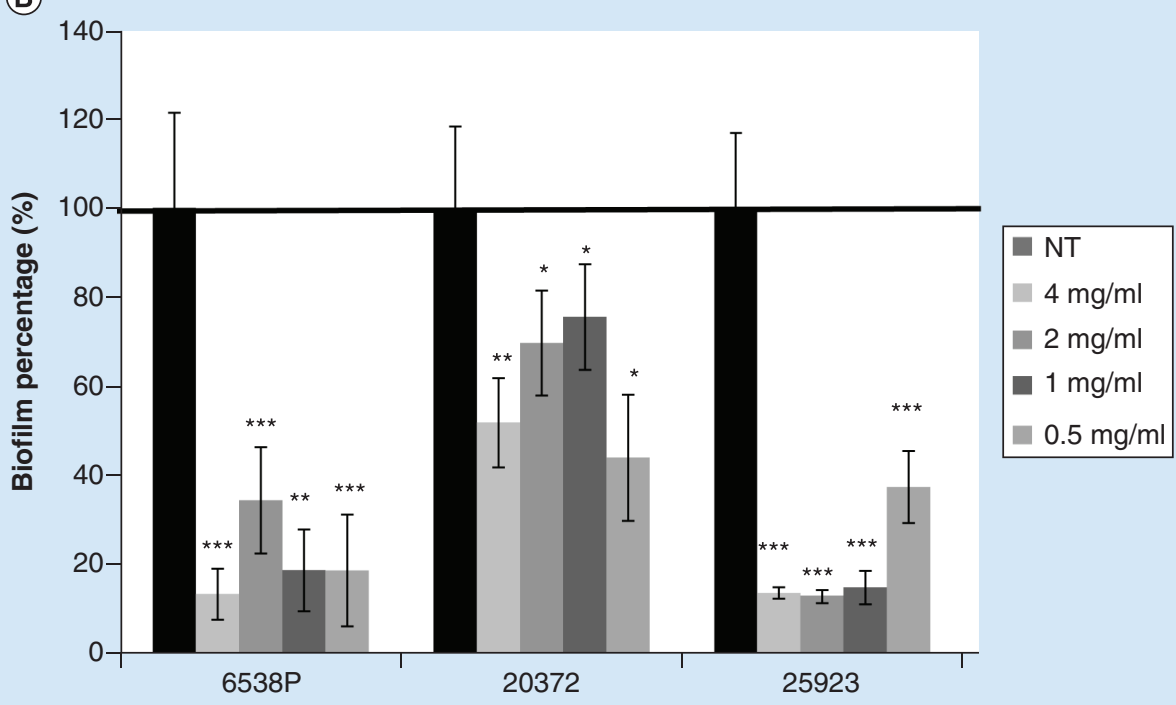

Figure 2. IN_TAA207 (A) and IN_TAD1S (B) extracts on biofilm formation of different $S$. aureus strains. Serial dilutions of each extract starting from $4 \mathrm{mg} / \mathrm{ml}$ were evaluated. Data are reported as the percentage of residual biofilm. Each data point represents the mean \pm SD of three independent samples. Differences in mean absorbance were compared with the untreated control (NT, black bar) and considered significant when $p<0.05$ according to Student's $t$-test. ${ }^{*} \mathrm{p}<0.05 ; * * \mathrm{p}<0.01 ; * * * \mathrm{p}<0.001$.

NT: Not-treated; SD: Standard deviation.

activity on S. aureus strain 6538P (Figure 3A). Results showed strong antibiofilm activity in fraction 3, eluted with only chloroform. This sample was analyzed by GC-MS revealing the presence of saturated and unsaturated long-chain fatty acids as follows: C14:0, C14:1, C16:0, C16:1, C18:1, and a very low amount of C18:0 (Figure 3B).

Antibiofilm activity of fractions derived from chromatographic separation of IN_TAD1S was also evaluated, as reported in Figure 3C. In this case, different fractions were able to inhibit biofilm formation of $S$. aureus 6538P. The strongest effect was observed for fractions 5, 8, 11 (eluted with chloroform) and 20 (eluted with the chloroform/methanol 99:1). Moreover, for these fractions, an antibacterial activity has been observed.

To obtain more information about the chemical composition, each fraction was analyzed by GC-MS. Also, in this case, chromatograms revealed the presence of saturated and unsaturated long-chain fatty acids, with slight differences in the relative amount of each chemical species (Supplementary Figure 1). A comparison between 


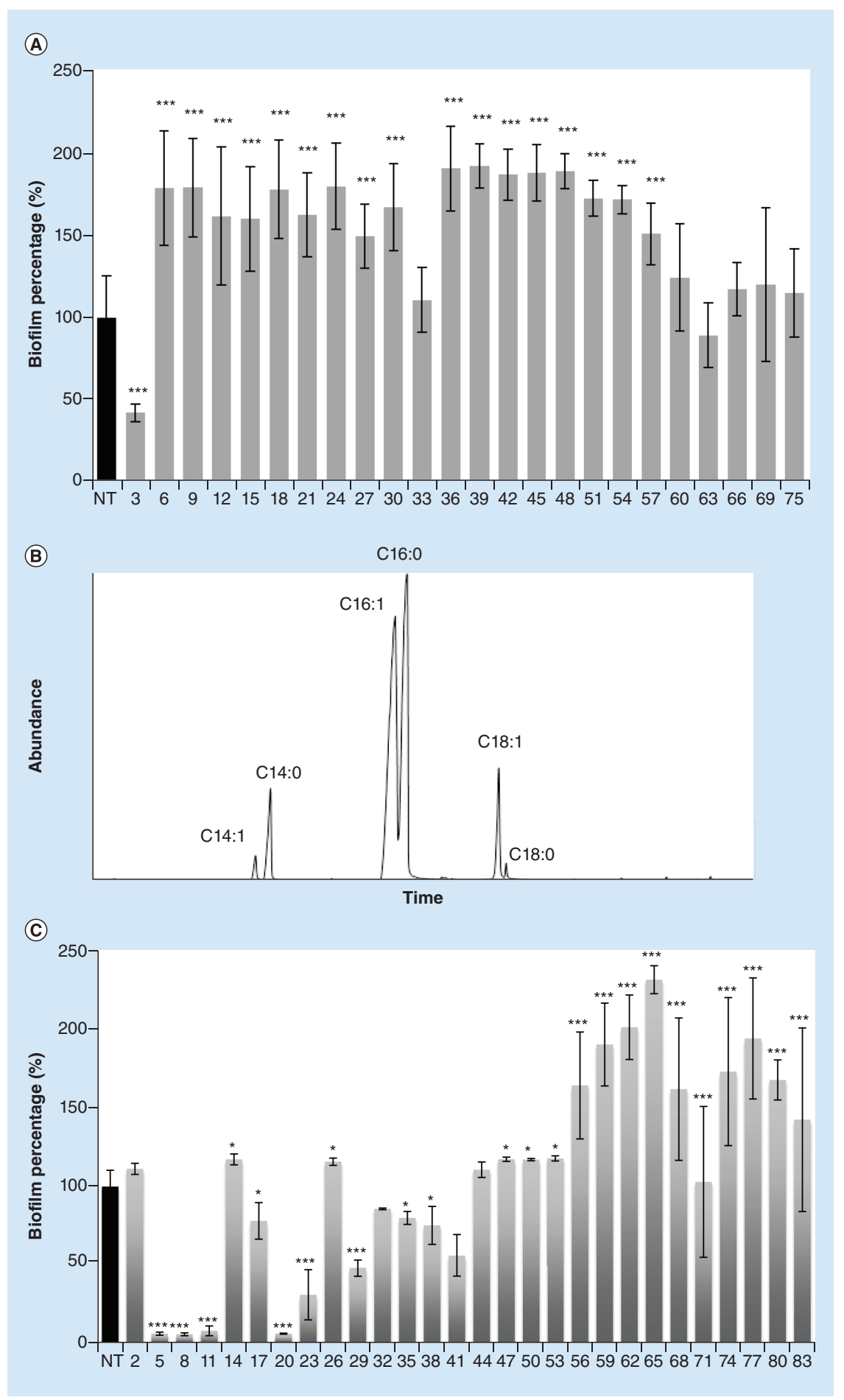

Figure 3. Chromatographic fractions from IN_TAA207 (A) and IN_TAD1S (C) on biofilm formation of S. aureus 6538P. Chromatographic profile of fraction 3 from IN_TAA207 (B). Each fraction was used at a concentration of $250 \mu \mathrm{g} / \mathrm{ml}$. Data are reported as the percentage of residual biofilm. Each data point represents the mean \pm SD of three different samples. Biofilm formation was considered unaffected in the range of 90-100\%. Differences in mean absorbance were compared with the untreated control and considered significant when $p<0.05$ according to Student's $t$-test. ${ }^{*} p<0.05 ; * * p<0.01 ; * * * p<0.001$.

SD: Standard deviation. 


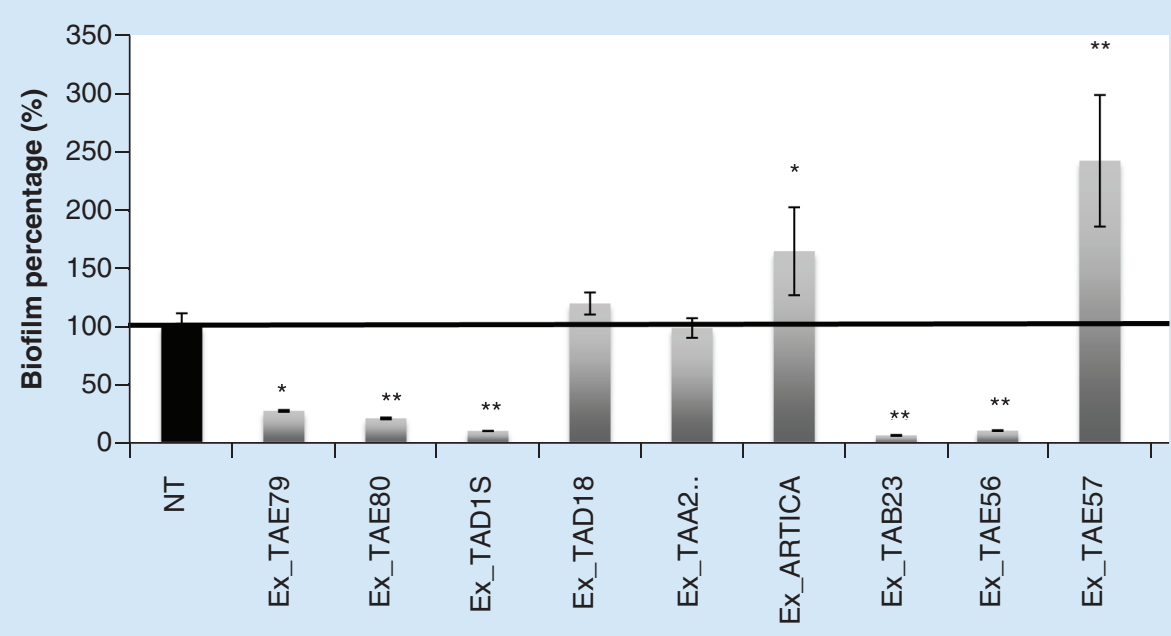

Figure 4. Extracellular extracts from cold-adapted bacteria on S. aureus 6538P biofilm formation. Data are reported as the percentage of residual biofilm after the treatment. Each data point represents the mean \pm SD of five independent samples. Differences in mean absorbance were compared with the untreated control (NT, black bar) and considered significant when $\mathrm{p}<0.05$ according to Student's $t$-test.

${ }^{*} \mathrm{p}<0.05 ; * * \mathrm{p}<0.01 ; * * * \mathrm{p}<0.001$.

NT: Not-treated; SD: Standard deviation.

IN_TAA207 and IN_TAD1S composition profiles revealed the presence in IN_TAD1S extract of fatty acids with an odd number of carbon atoms (i.e., C17:1; Supplementary Figure 1).

\section{Screening of antibiofilm activity of extracellular organic extracts against $S$. aureus}

Figure 4 shows the results obtained on $S$. aureus 6538P biofilm formation in the presence of different extracellular extracts from polar bacteria. Several extracts induced a strong reduction in biofilm formation by S. aureus 6538P. EX_TAE56 and EX_TAE57 were tested at a concentration of $0.5 \mathrm{mg} / \mathrm{ml}(1 / 2 \mathrm{MIC}$ value), considering their antimicrobial activity reported in Table 2 . Active extracts were selected for further analysis and tested on biofilm formation of three different $S$. aureus strains (65385P, 20372 and 25923). Furthermore, an assessment of the activity at different concentrations suggested a dose-dependent effect (Figure 5). Best inhibition in biofilm formation was obtained using EX_TAD1S on all tested strains (Figure 5C), indeed the percentage of residual biofilm was lower than $40 \%$, even at a lower concentration of the extract $(0.5 \mathrm{mg} / \mathrm{ml})$. For this reason, EX_TAD1S was chosen for further analysis.

\section{Fractionation of extracellular extract from Pseudomonas sp. TAD1S}

EX_TAD1S was analyzed by ${ }^{1} \mathrm{H}$ NMR. This latter suggests the presence of a complex mixture of compounds (data not shown). To isolate the active molecule/s from EX_TAD1S, a preliminary purification step on a silica gel column was carried out, as reported in the Materials \& methods section. The obtained fractions were tested to evaluate the antibiofilm activity on $S$. aureus (Figure 6A). The results clearly indicated the presence of the active molecule $/ \mathrm{s}$ in fractions 15 and 17 (eluted with chloroform/methanol 8:2). The ${ }^{1} \mathrm{H}$ NMR spectra showed a profile almost identical for these two fractions. The analysis of the spectrum of fraction 15, reported in Figure 6B, suggested the presence of hydrocarbon chains, as indicated by the signals in the range of $\delta 0.5-1.5 \mathrm{ppm}$. In addition, signals appearing between $\delta 6.4$ and $8.8 \mathrm{ppm}$ strongly supported $\mathrm{N}-\mathrm{H}$ protons (Figure $6 \mathrm{~B}$ ) that together with signals around $\delta 4 \mathrm{ppm}$, indicated a peptide backbone. All together, these signals indicated the lipopeptide nature of the active compound [30-32]. The GC-MS analysis of products obtained after the sample methanolysis followed by acetylation revealed the presence of the following amino acids: serine, valine, leucine and glutamic acid. This aminoacidic composition suggests a structure like those usually found for lipopeptides isolated from Bacillus and Pseudomonas species [33]. In addition, GC-MS analysis suggested that the lipopeptide was constituted by various isoforms, due to the difference in the length of the fatty acids, since it revealed a mixture of C15:0, C16:0, C16:1, C17:0, C18:0 and C18:1. Finally, the absence in the GC-MS chromatogram of peaks attributable to $\beta$-hydroxylated fatty acids 

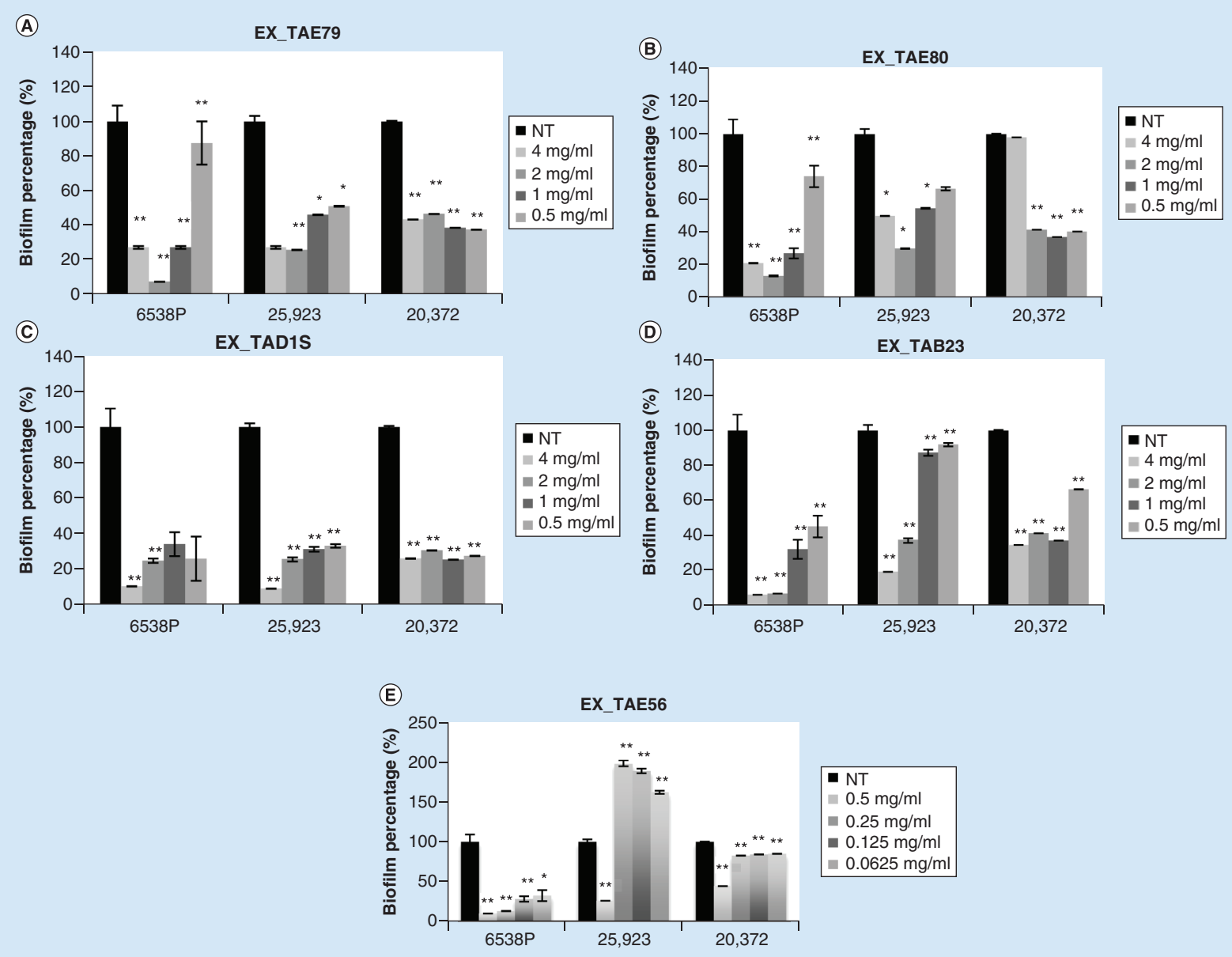

Figure 5. Selected extracellular extracts on biofilm formation of different $S$. aureus strains. Biofilm formation was evaluated after incubation of serial dilutions of extracts starting from $4 \mathrm{mg} / \mathrm{ml}$ (EX_TAE56 was tested starting from $\frac{1}{2}$ MIC value corresponding to $1 \mathrm{mg} / \mathrm{ml}$ ). Data are reported as the percentage of residual biofilm. Each data point represents the mean \pm SD of three independent samples. Differences in mean absorbance were compared with the untreated control (NT, black bar) and considered significant when $\mathrm{p}<0.05$ according to Student $t$-test.

${ }^{*} \mathrm{p}<0.05 ; * * \mathrm{p}<0.01 ; * * * \mathrm{p}<0.001$.

NT: Not-treated; SD: Standard deviation.

suggested a cyclic structure with a terminal amide-linked fatty acid [34]. We also evaluated the antimicrobial activity of this fraction on $S$. aureus $6538 \mathrm{P}$ that was found at a concentration of $400 \mu \mathrm{g} / \mathrm{ml}$.

\section{Discussion}

The investigation of new unexplored habitats and uncommon environments has become an important source for the discovery of novel bacterial metabolites with antimicrobial activity [35]. Since the antimicrobial activity of microorganisms living in extreme environments has not been explored as widely as for mesophilic microorganisms, the traditional approach of isolating and cultivating new microorganisms from underexplored habitats can be productive.

In this paper, polar marine bacteria belonging to Pseudoalteromonas, Pseudomonas and Psychromonas genera were grown in a synthetic medium and organic extracts of intracellular and from extracellular contents were analyzed to identify novel antibiofilm/antimicrobial molecules. The MIC evaluation against $S$. aureus $6538 \mathrm{P}$ revealed that the samples did not possess a relevant antimicrobial activity, while some of them strongly prevented biofilm formation by $S$. aureus 6538 P.

IN_TAA207 and IN_TAD1S revealed a similar antibiofilm activity but were both selected because a preliminary analysis indicated that their chemical composition was different (data not shown). The samples were tested against 


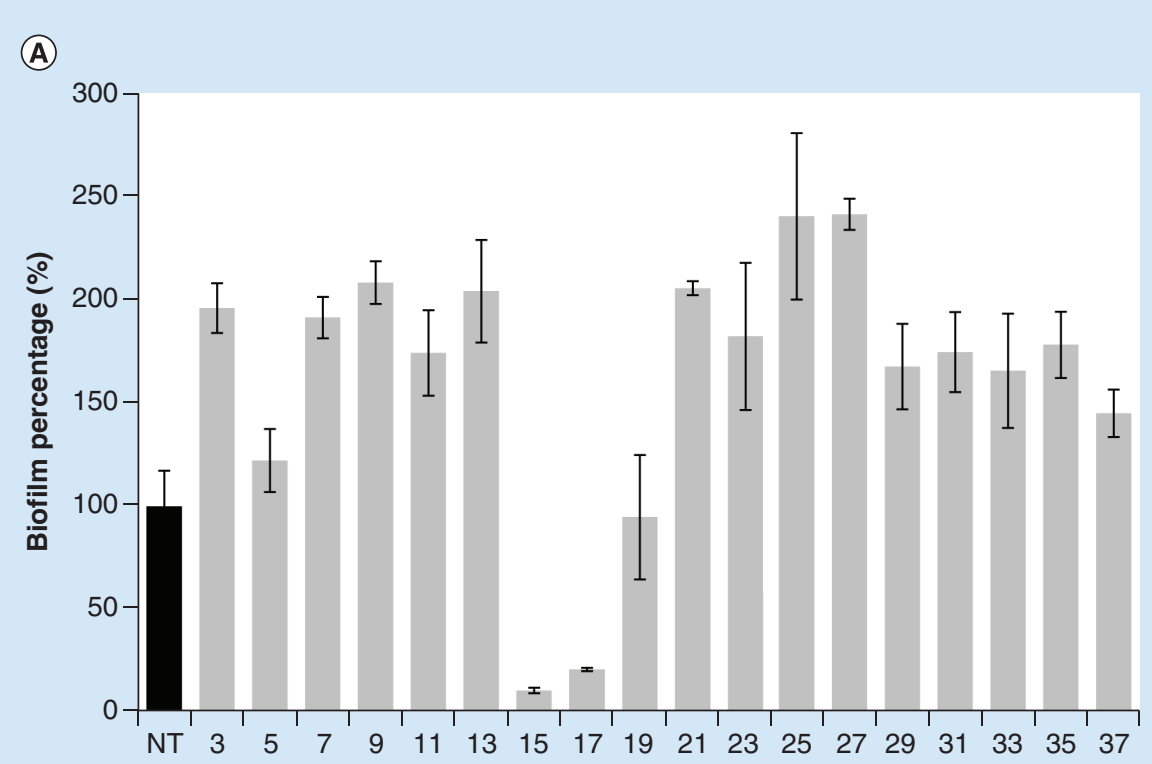

(B)

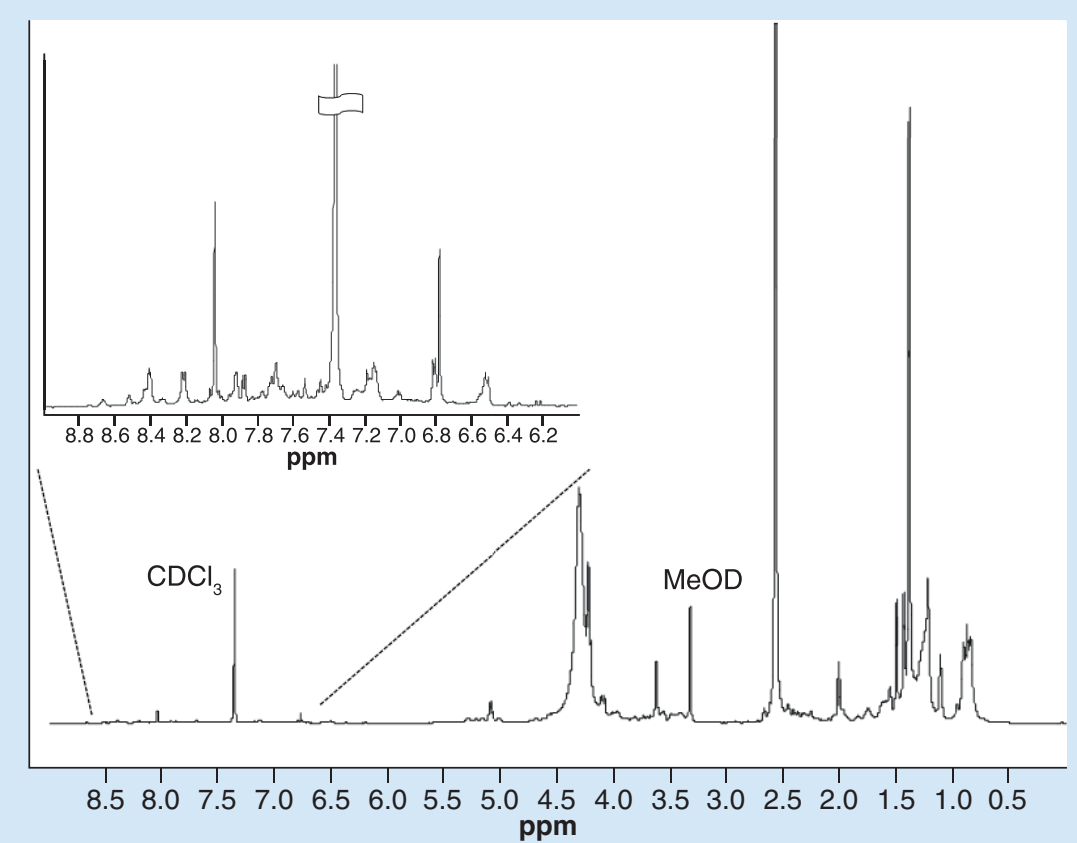

Figure 6. Chromatographic fractions from EX_TAD1S against $S$. aureus 6538P $(\mathrm{A})$ and ${ }^{1} \mathrm{H}$ NMR spectrum of the active fraction (B). Each fraction was used at a concentration of $250 \mu \mathrm{g} / \mathrm{ml}$. Data are reported as the percentage of residual biofilm. Each data point represents the mean \pm SD of three different samples. Biofilm formation was considered unaffected in the range of $90-100 \%$. Differences in mean absorbance were compared with the untreated control and considered significant when $\mathrm{p}<0.05$ according to Student's $t$-test. (B) ${ }^{1} \mathrm{H}$ NMR spectrum of the active fraction 15 from EX_TAD1S. Spectrum was recorded in $\mathrm{CDCl}_{3}: \mathrm{CD}_{3} \mathrm{OD}$ at $600 \mathrm{MHz}$ and $298 \mathrm{~K}$.

${ }^{*} \mathrm{p}<0.05 ; * * \mathrm{p}<0.01 ; * * * \mathrm{p}<0.001$

SD: Standard deviation.

three different $S$. aureus strains: ATCC 6538P and ATCC 25923 are both reference strains for antimicrobial testing, while ATCC 20372 is a clinical isolate. These strains show a different ability to form biofilm: ATCC 25923 is classified as a strong biofilm producer, ATCC 6538P is a medium/strong biofilm producer and ATCC 20372 is considered a medium/weak biofilm producer [36]. IN_TAA207 and IN_TAD1S were both able to inhibit biofilm formation of all tested S. aureus strains, and the effect of IN_TAA207 was clearly dose dependent, whereas 
the activity of IN_TAD1S was independent of the extract concentration. The different chemical composition of IN_TAA207 and IN_TAD1S extracts and the different features of the three tested $S$. aureus strains could explain the reported results. Both extracts were fractionated, and the resulting fractions were tested to assess their antibiofilm activity. The chromatographic fraction 3, obtained from IN_TAA207 extract, and chromatographic fractions 5, 8, 11 and 20 from IN_TAD1S extract showed the best antibiofilm activity. Moreover, all fractions from IN_TAD1S with antibiofilm action showed antimicrobial activity. All these fractions contained saturated and unsaturated long-chain fatty acids. Free fatty acids are ubiquitous on the surface of human skin and represent the predominant components in human sebum [37]. Particularly, medium- to long-chain fatty acids (C8 to C18) display antibacterial activity against a broad range of Gram-positive bacteria and are considered responsible for at least part of the direct antimicrobial activity of the skin surface against pathogen colonization and infection [38]. The antibacterial efficacy of fatty acids stems from their membrane-destabilizing activity that causes increased cell permeability and cell lysis.

Some of the identified fatty acids were common to different active fractions; in detail, either fraction 3 from IN_TAA207 and fractions 5, 8, 11 from IN_TAD1S contained C16:1 (palmitoleic), C16:0 and C18:1 (oleic acid). For these fatty acids, the antibacterial activity against $S$. aureus was previously observed [39-41]. Two odd long fatty acids, C17:1 and C19:1, were identified in fractions 5, 8, 11 and 20 from IN_TAD1S. Although several bacteria produce odd-numbered fatty acid [42,43], to the best of our knowledge, no information about their possible antimicrobial activity against $S$. aureus was previously recorded. Future studies will be aimed to clarify if these molecules contribute to the antimicrobial activity of IN_TAD1S fractions and if they can be considered responsible for the differences in the activity of IN_TAD1S and IN_TAA207 extracts. Anyway, the reported antibiofilm activity of IN_TAD1S and IN_TAA207 was found at sub-MIC concentrations.

The screening of polar extracellular extracts revealed that EX_TAE79, EX_TAE80 EX_TAB23, EX_TAD1S and EX_TAE56 were able to affect $S$. aureus biofilm formation. The attention was focused on EX_TAD1S since this sample efficiently impairs biofilm formation in all three tested strains, with a percentage of residual biofilm lower than $40 \%$ as compared with the untreated sample. Also, in this case, the extract was fractionated, and the corresponding fractions were analyzed. Fractions 15 and 17 displayed a high level of activity against $S$. aureus biofilm (Figure 6A). The ${ }^{1} \mathrm{H}$ NMR spectrum of fraction 15 (Figure 6B) indicated the presence of N-H (6.4$8.6 \mathrm{ppm})$ and $\mathrm{C}-\mathrm{H}(4.5-5.5 \mathrm{ppm})$ signals that, together with signals of a long aliphatic chain $(0.5-1.5 \mathrm{ppm})$ suggested the lipopeptide nature of the active compound [30-32]. The GC-MS analysis revealed the presence of serine, leucine, valine and glutamic acid, an amino acid composition agreed with the compositions obtained for other lipopeptides [30,31], moreover this analysis suggests a cyclic structure of this lipopeptide.

Lipopeptides are produced by several bacterial species; they are amphiphilic molecules consisting of short linear chains or cyclic structures of amino acids, linked to a fatty acid via ester or amide bonds or both. They are proved to act as antibiotics, antiadhesives, antitumor compounds and foaming agents [44-48]. Very few examples of lipopeptides isolated from cold-adapted bacteria were reported. Psendomonas fluorescens BD5, a bacterium isolated from Archipelago of Svalbard, produces a cyclic lipopeptide named pseudofactin [49] endowed with a very good emulsification activity and with antiadhesive activity against several pathogenic microorganisms (Escherichia coli, Enterococcus faecalis, Enterococcus hirae, S. epidermidis, Proteus mirabilis and Candida albicans) [49]. Another coldadapted bacterium able to produce lipopeptides is Bacillus amyloliquefaciens Pc3 that was isolated from Antarctic seawater [50].

Although in the literature few publications describe lipopeptides from cold-adapted bacteria, many reports describe the ability of lipopeptides to kill pathogenic bacteria. Interestingly, several lipopeptides are active against S. aureus. Some examples are Daptomycin, a cyclic lipopeptide, that was approved as an antibiotic by the US FDA in 2003 for the treatment of complicated skin [51]; brevibacillin, a lipopeptide produced by a strain of Brevibacillus laterosporus showing antimicrobial activity against $S$. aureus [52].

\section{Conclusion}

The cyclic lipopeptide isolated from Pseudomonas sp. TAD1S does not display antimicrobial activity on $S$. aureus (MIC resulted to be $400 \mu \mathrm{g} / \mathrm{ml}$ ) but it resulted to be an effective antibiofilm molecule able to strongly reduce the $S$. aureus biofilm formation. Furthermore, preliminary results indicate that it also works as a biosurfactant (Supplementary Figure 2). 


\section{Future perspective}

The new cyclic lipopeptide isolated from Antarctic bacterium Pseudomonas sp. TAD1S may be potentially useful in a wide variety of biotechnological and medical applications. Further studies are needed to better define its chemical structure and to explore its biological activity and applicability as an anti-infective agent.

\section{Summary points}

Among bacteria, staphylococci are recognized as the most frequent causes of biofilm-associated infections

- Staphylococcus aureus is often present asymptomatically on districts of the human body.

- S. aureus possesses a collection of virulence factors and displays the ability to acquire resistance to most antibiotics.

- The antibiotic resistance of $S$. aureus is related, in most cases, to its ability to develop a protective architecture called biofilm.

Unexplored habitats \& uncommon environments can be considered an important source for the discovery of novel bacterial metabolites with antimicrobial activity

- Marine microorganisms are considered potential sources for the discovery of new metabolically active molecules.

- Some of these bioactive molecules have already been selected for the treatment of various diseases and many of them are under clinical investigations.

- Cold-adapted marine bacteria deriving from polar regions represent an untapped reservoir of biodiversity endowed with an interesting chemical repertoire.

- Intracellular and extracellular organic extracts obtained from cultures of polar marine bacteria were tested against $S$. aureus.

Conclusion

- Identification of a lipopeptide able to strongly reduce the $S$. aureus biofilm formation with antimicrobial activity at high concentration.

- The new cyclic lipopeptide isolated from Antarctic bacterium Pseudomonas sp. TAD1S may be potentially useful in a wide variety of biotechnological and medical applications.

- Our findings indicate that the lipopeptide also works as a biosurfactant.

\section{Supplementary data}

To view the supplementary data that accompany this paper please visit the journal website at: www.futuremedicine.com/doi/full/ 10.2217/fmb-2019-0147

Author contributions

Design of the work, acquisition of data, analysis and interpretation, drafting and critical revision, approval of the final version and agreement to be accountable for the work were performed by all the authors.

Financial \& competing interests disclosure

The authors have no relevant affiliations or financial involvement with any organization or entity with a financial interest in or financial conflict with the subject matter or materials discussed in the manuscript. This includes employment, consultancies, honoraria, stock ownership or options, expert testimony, grants or patents received or pending, or royalties.

No writing assistance was utilized in the production of this manuscript.

\section{References}

Papers of special note have been highlighted as: $\bullet$ of interest; $\bullet \bullet$ of considerable interest

1. Katz ML, Mueller LV, Polyakov M, Weinstock SF. Where have all the antibiotic patents gone? Nat. Biotechnol. 24, 1529-1531 (2006).

2. Hall CW, Mah TF. Molecular mechanisms of biofilm-based antibiotic resistance and tolerance in pathogenic bacteria. FEMS Microbiol. Rev. 41, 276-301 (2017).

-• Description of mechanisms of biofilm-based antibiotic resistance in pathogenic bacteria.

3. Otto M. Staphylococcal biofilms. Curr. Top. Microbiol. Immunol. 322, 207-228 (2008).

4. Lakhundi S, Zhang K. Methicillin-resistant Staphylococcus aureus: molecular characterization, evolution, and epidemiology. Clin. Microbiol. Rev. 31, pii:e00020-18 (2018).

-• Shows the evolution of methicillin-resistant Staphylococcus aureus.

5. Hall-Stoodley L, Stoodley P. Evolving concepts in biofilm infections. Cell. Microbiol. 11, 1034-1043 (2009). 
6. Liu W, Li J, Cheng M et al. A surface-engineered polyetheretherketone biomaterial implant with direct and immunoregulatory antibacterial activity against methicillin-resistant Staphylococcus aureus. Biomaterials 208, 8-20 (2019).

7. Talbot GH, Bradley J, Edwards JE Jr, Gilbert D, Scheld M, Bartlett JG. Antimicrobial Availability Task Force of the Infectious Diseases Society of America. Bad bugs need drugs: an update on the development pipeline from the Antimicrobial Availability Task Force of the Infectious Diseases Society of America. Clin. Infect. Dis. 42, 657-668 (2006).

8. Artini M, Papa R, Barbato G et al. Bacterial biofilm formation inhibitory activity revealed for plant derived natural compounds. Bioorg. Med. Chem. 20, 920-926 (2012).

9. Manivasagan P, Kang KH, Sivakumar K, Li-Chan EC, Oh HM, Kim SK. Marine actinobacteria: an important source of bioactive natural products. Environ. Toxicol. Pharmacol. 38, 172-188 (2014).

10. Mehbub MF, Lei J, Franco C, Zhang W. Marine sponge derived natural products between 2001 and 2010: trends and opportunities for discovery of bioactives. Mar. Drugs 12, 4539-4577 (2014).

11. Artini M, Patsilinakos A, Papa R et al. Antimicrobial and antibiofilm activity and machine learning classification analysis of essential oils from different Mediterranean plants against Pseudomonas aeruginosa. Molecules 23, pii:E482 (2018).

12. Newman DJ, Cragg GM, Snader KM. Natural products as sources of new drugs over the period 1981-2002. J. Nat. Prod. 66, 1022-1037 (2003).

13. Katz L, Baltz RH. Natural product discovery: past, present, and future. J. Ind. Microbiol. Biotechnol. 43, 155-176 (2016).

14. Bhatnagar I, Kim SK. Immense essence of excellence: marine microbial bioactive compounds. Mar. Drugs 8, 2673-2701 (2010).

15. Ng TB, Cheung RC, Wong JH, Bekhit AA, Bekhit Ael-D. Antibacterial products of marine organisms. Appl. Microbiol. Biotechnol. 99, 4145-4173 (2015).

-. An overview of new antimicrobials isolated from marine organisms.

16. Molinski TF, Dalisay DS, Lievens SL, Saludes JP. Drug development from marine natural products. Nat. Rev. Drug. Discov. 8, 69-85 (2009).

17. Liu Y. Renaissance of marine natural product drug discovery and development. J. Mari. Sci. Res. Dev. 2, e106 (2012).

18. Lo Giudice A, Bruni V, Michaud L. Characterization of Antarctic psychrotrophic bacteria with antibacterial activities against terrestrial microorganisms. J. Basic Microbiol. 47, 496-505 (2007).

19. Núñez-Montero K, Barrientos L. Advances in Antarctic research for antimicrobial discovery: a comprehensive narrative review of bacteria from Antarctic environments as potential sources of novel antibiotic compounds against human pathogens and microorganisms of industrial importance. Antibiotics (Basel) 7, pii:E90 (2018).

-. An overview of new antimicrobials isolated from Antarctic bacteria.

20. Neifar M, Maktouf S, Ghorbel RE, Jaouani A, Cherif A. Extremophiles as a source of novel bioactive compounds with industrial potential. In: Biotechnology of Bioactive Compounds: Sources and Applications. John Wiley \& Sons, Chichester, West Sussex, UK (2015).

21. Papa R, Parrilli E, Sannino F et al. Anti-biofilm activity of the Antarctic marine bacterium Pseudoalteromonas haloplanktis TAC125. Res. Microbiol. 164, 450-456 (2013).

22. Parrilli E, Papa R, Carillo S et al. Anti-biofilm activity of Pseudoalteromonas haloplanktis TAC125 against Staphylococcus epidermidis biofilm: evidence of a signal molecule involvement? Int. J. Immunopathol. Pharmacol. 28, 104-113 (2015).

23. Casillo A, Papa R, Ricciardelli A et al. Anti-biofilm activity of a long-chain fatty aldehyde from Antarctic Pseudoalteromonas haloplanktis TAC125 against Staphylococcus epidermidis biofilm. Front. Cell. Infect. Microbiol. 7, 46 (2017).

- Describes a new small antibiofilm molecule isolated from an Antarctic bacterium.

24. Papaleo MC, Romoli R, Bartolucci G et al. Bioactive volatile organic compounds from Antarctic (sponges) bacteria. N. Biotechnol. 30, 824-838 (2013).

25. Sannino F, Parrilli E, Apuzzo GA et al. Pseudoalteromonas haloplanktis produces methylamine, a volatile compound active against Burkholderia cepacia complex strains. N. Biotechnol. 35, 13-18 (2017).

26. Papa R, Selan L, Parrilli E et al. Anti-biofilm activities from marine cold adapted bacteria against Staphylococci and Pseudomonas aeruginosa. Front. Microbiol. 6, 1333 (2015).

- Shows the antibiofilm activities of several polar bacteria against different pathogens.

27. CLSI Supplement M100. Performance Standards for Antimicrobial Susceptibility Testing (27th Edition). Clinical and Laboratory Standards Institute, Wayne, PA, USA (2017).

28. Busetti A, Shaw G, Megaw J, Gorman SP, Maggs CA, Gilmore BF. Marine-derived quorum-sensing inhibitory activities enhance the antibacterial efficacy of tobramycin against Pseudomonas aeruginosa. Mar. Drugs 13, 1-28 (2014).

29. Artini M, Papa R, Scoarughi GL et al. Comparison of the action of different proteases on virulence properties related to the staphylococcal surface. J. Appl. Microbiol. 114, 266-277 (2013).

30. Liu XY, Yang SZ, Mu BZ. Production and characterization of a C15-surfactin-O-methyl ester by a lipopeptide producing strain Bacillus subtilis HSO121. Process Biochem. 44, 1144-1151 (2009). 
31. Naruse N, Tenmyo O, Kobaru S et al. Pumilacidin, a complex of new antiviral antibiotics production, isolation, chemical properties, structure and biological activity. J. Antibiot. 43, 267-280 (1990).

32. Saggese A, Culurciello R, Casillo A, Corsaro MM, Ricca E, Baccigalupi L. A marine isolate of Bacillus pumilus secretes a pumilacidin active against Staphylococcus aureus. Mar. Drugs 16, 180 (2018).

33. Inès M, Dhouha G. Lipopetide surfactants: production, recovery and pore forming capacity. Peptides 71, 100-112 (2015).

34. Janek T, Łukaszewicz M, Rezanka T, Krasowska A. Isolation and characterization of two new lipopeptide biosurfactants produced by Pseudomonas fluorescens BD5 isolated from water from the Arctic Archipelago of Svalbard. Bioresour. Technol. 101, 6118-6123 (2010).

35. Donadio S, Maffioli S, Monciardini P, Sosio M, Jabes D. Antibiotic discovery in the twenty-first century: current trends and future perspectives. J. Antibiot. 63, 423-430 (2010).

36. Cafiso V, Bertuccio T, Santagati M et al. agr-Genotyping and transcriptional analysis of biofilm-producing Staphylococcus aureus. FEMS Immunol. Med. Microbiol. 51, 220-227 (2007).

37. Wille JJ, Kydonieus A. Palmitoleic acid isomer (C16:1delta6) in human skin sebum is effective against Gram-positive bacteria. Skin Pharmacol. Appl. Skin Physiol. 16, 176-187 (2003).

38. Drake DR, Brogden KA, Dawson DV, Wertz PW. Thematic review series: skin lipids. Antimicrobial lipids at the skin surface. J. Lipid Res. 49, 4-11 (2008).

39. Kabara JJ, Swieczkowski DM, Conley AJ, Truant JP. Fatty acids and derivatives as antimicrobial agents. Antimicrob. Agents Chemother. 2, 23-28 (1972).

40. Kitahara T, Koyama N, Matsuda J et al. Antimicrobial activity of saturated fatty acids and fatty amines against methicillin-resistant Staphylococcus aureus. Biol. Pharm. Bull. 27, 1321-1326 (2004).

41. Kelsey JA, Bayles KW, Shafii B, McGuire MA. Fatty acids and monoacylglycerols inhibit growth of Staphylococcus aureus. Lipids 41, 951-961 (2006).

42. Kamimura K, Fuse H, Takimura O, Yamaoka Y, Ohwada K, Hashimoto J. Pressure-induced alteration in fatty acid composition of barotolerant deep-sea bacterium. J. Oceanogr. 48, 104 (1992).

43. Ponder MA, Gilmour SJ, Bergholz PW et al. Characterization of potential stress responses in ancient Siberian permafrost psychroactive bacteria. FEMS Microbiol. Ecol. 53, 103-115 (2005).

44. Das P, Mukherjee S, Sivapathasekaran C, Sen R. Microbial surfactants of marine origin: potentials and prospects. Adv. Exp. Med. Biol. 672, 88-101 (2010).

45. Banat IM, Franzetti A, Gandolfi I et al. Microbial biosurfactants production, applications and future potential. Appl. Microbiol. Biotechnol. 87, 427-444 (2010).

46. Biniarz P, Eukaszewicz M, Janek T. Screening concepts, characterization and structural analysis of microbial-derived bioactive lipopeptides: a review. Crit. Rev. Biotechnol. 8551, 1-18 (2016).

- An overview of microbial-derived bioactive lipopeptides.

47. Nielsen TH, Nybroe O, Koch B, Hansen M, Sørensen J. Genes involved in cyclic lipopeptide production are important for seed and straw colonization by Pseudomonas sp. strain DSS73. Appl. Environ. Microbiol. 71, 4112-4116 (2005).

48. Raaijmakers JM, de Bruijn I, de Kock MJ. Cyclic lipopeptide production by plant-associated Pseudomonas spp.: diversity, activity, biosynthesis, and regulation. Mol. Plant Microbe Interact. 19, 699-710 (2006).

49. Janek T, Łukaszewicz M, Krasowska A. Antiadhesive activity of the biosurfactant pseudofactin II secreted by the Arctic bacterium Pseudomonas fluorescens BD5. BMC Microbiol. 12, 24 (2012).

50. Ding L, Zhang S, Guo W, Chen X. Exogenous indole regulates lipopeptide biosynthesis in Antarctic Bacillus amyloliquefaciens Pc3.J. Microbiol. Biotechnol. 28, 784-795 (2018).

51. Marty FM, Yeh WW, Wennersten CB et al. Emergence of a clinical daptomycin-resistant Staphylococcus aureus isolate during treatment of methicillin-resistant Staphylococcus aureus bacteremia and osteomyelitis. J. Clin. Microbiol. 44, 595-597 (2006).

52. Yang X, Huang E, Yuan C, Zhang L, Yousef AE. Isolation and structural elucidation of brevibacillin: an antimicrobial lipopeptide from Brevibacillus laterosporus that combats drug-resistant Gram-positive bacteria. Appl. Environ. Microbiol. 82, 2763-2772 (2016).

- Describes the antimicrobial activity of a natural lipopeptide against drug-resistant Gram-positive bacteria.

53. Groudieva T, Grote R, Antranikian G. Psychromonas arctica sp. nov., a novel psychrotolerant, biofilm-forming bacterium isolated from Spitzbergen. Int. J. Syst. Evol. Microbiol. 53, 539-545 (2003).

54. Feller G, D’Amico S, Benotmane AM, Joly F, Van Beeumen J, Gerday C. Characterization of the C-terminal propeptide involved in bacterial wall spanning of alpha-amylase from the psychrophile Alteromonas haloplanktis. J. Biol. Chem. 273, 12109-12115 (1998). 